\title{
Revealing laser crystallization mechanism of silicon thin films via pulsed IR lasers
}

\author{
Kamil Çınar, ${ }^{1,2}$ Cihan Yeşil, ${ }^{1}$ and Alpan Bek ${ }^{1,2}$ \\ ${ }^{1}$ Department of Physics, Middle East Technical University, Ankara, Turkey \\ ${ }^{2}$ The Center for Solar Energy Research and Applications (GÜNAM), Middle East \\ Technical University, Ankara, Turkey
}

\section{Supplementary Information}

Here, we provide supplementary information on details of simulation parameters and variables implemented in our model. We also provide detailed explanation to the pulse-to-pulse displacement. Additionally, we also provide a simulation of temporal temperature behavior in order to present an opinion on crack-like interfacial deformations.

\section{Simulation Parameters}

$$
\begin{aligned}
& h=30\left[\mathrm{~W} /\left(\mathrm{m}^{2} . \mathrm{K}\right)\right] \text { "Heat transfer coefficient" } \\
& T_{0}=300[\mathrm{~K}] \text { "Initial temperature" } \\
& T_{m, a}=1560[\mathrm{~K}] \text { "Melting point of a-Si" } \\
& T_{m, c}=1685[\mathrm{~K}] \text { "Melting point of c-Si" } \\
& T_{b}=3538[\mathrm{~K}] \text { "Boiling point of Si" } \\
& \rho_{l}=2.57\left[\mathrm{~g} / \mathrm{cm}^{3}\right] \text { "Density of I-Si" } \\
& \rho_{a}=2.32\left[\mathrm{~g} / \mathrm{cm}^{3}\right] \text { "Density of a-Si" } \\
& \rho_{c}=2.329\left[\mathrm{~g} / \mathrm{cm}^{3}\right] \text { "Density of c-Si" } \\
& \Delta H_{a}=1320[\mathrm{~J} / \mathrm{g}] \text { "Latent heat of a-Si" } \\
& \Delta H_{c}=1800[\mathrm{~J} / \mathrm{g}] \text { "Latent heat of c-Si" } \\
& k_{l}=110[\mathrm{~W} /(\mathrm{m} . \mathrm{K})] \text { "Thermal conductivity of I-Si" } \\
& k_{a}=2[\mathrm{~W} /(\mathrm{m} . \mathrm{K})] \text { "Thermal conductivity of a-Si" } \\
& k_{c}=145[\mathrm{~W} /(\mathrm{m} . \mathrm{K})] \text { "Thermal conductivity of c-Si" } \\
& C_{p, S i}=0.95 \text { [J/(g.K)] "Specific heat capacity of Si @1600 K" } \\
& C_{p, a s i}=0.925 \text { [J/(g.K)]" "Specific heat capacity of a-Si @ } 920 \text { K" } \\
& C_{p, I S i}=0.975[\mathrm{~J} /(\mathrm{g} . \mathrm{K})] \text { ]" "Specific heat capacity of I-Si above } 1680 \mathrm{~K} \text { " } \\
& C_{p, c S i}=0.97[\mathrm{~J} /(\mathrm{g} . \mathrm{K})] \text { " "Specific heat capacity of I-Si below } 1680 \mathrm{~K} \text { " } \\
& \alpha_{S i}=4.5 \times 10^{-6}\left[\mathrm{~K}^{-1}\right] \text { "Thermal expansion coefficient of Si @ } 1400 \mathrm{~K} " \\
& \mu_{l}=0.8[\mathrm{~Pa} . \mathrm{s}] \text { "Dynamic viscosity of I-Si" } \\
& \nu=1.1 \text { "Heat capacity ratio" }
\end{aligned}
$$


$f=100[\mathrm{kHz}]$ "Repetition rate"

$d_{f x}=45[\mu \mathrm{m}]$ "Focal width in $\mathrm{x}$ (Source term dimension in $\mathrm{x}$ )"

$d_{f y}=1250[\mu \mathrm{m}]$ "Focal length in y (Source term dimension in y)"

$\tau=200[\mathrm{~ns}]$ " "Pulse duration"

$S_{y}=4[\mathrm{~mm}]$ "Source position in y"

$S_{x}=0[\mathrm{~mm}]$ "Source start position in $\mathrm{x"}$

$Q_{f}=99.89[\%]$ "Overlap percentage"

$F_{A v}=0.21\left[\mathrm{~J} / \mathrm{cm}^{2}\right]$ "Average fluence per pulse"

$F_{p}=2 F_{A v}$ "Peak fluence per pulse"

$\varepsilon=0.45$ "Extinct energy ratio through Si film"

\section{Simulation Variables}

$v_{s}=f d_{w}\left(1-Q_{f} / 100\right) /\left(1-f \tau\left(1-Q_{f} / 100\right)\right)$ "Scan speed"

$I_{0}=\varepsilon F_{p}$ "Peak absorbed energy per pulse"

\section{Simulation Geometry}

Geometry is an $8 \mathrm{~mm}$ by $10 \mathrm{~mm}$ rectangular domain consisting a mesh of equilateral triangles with side length of $100 \mu \mathrm{m}$ (see Figure $S 1$ ). Boundaries and surfaces are made to satisfy the condition Eq. 4.

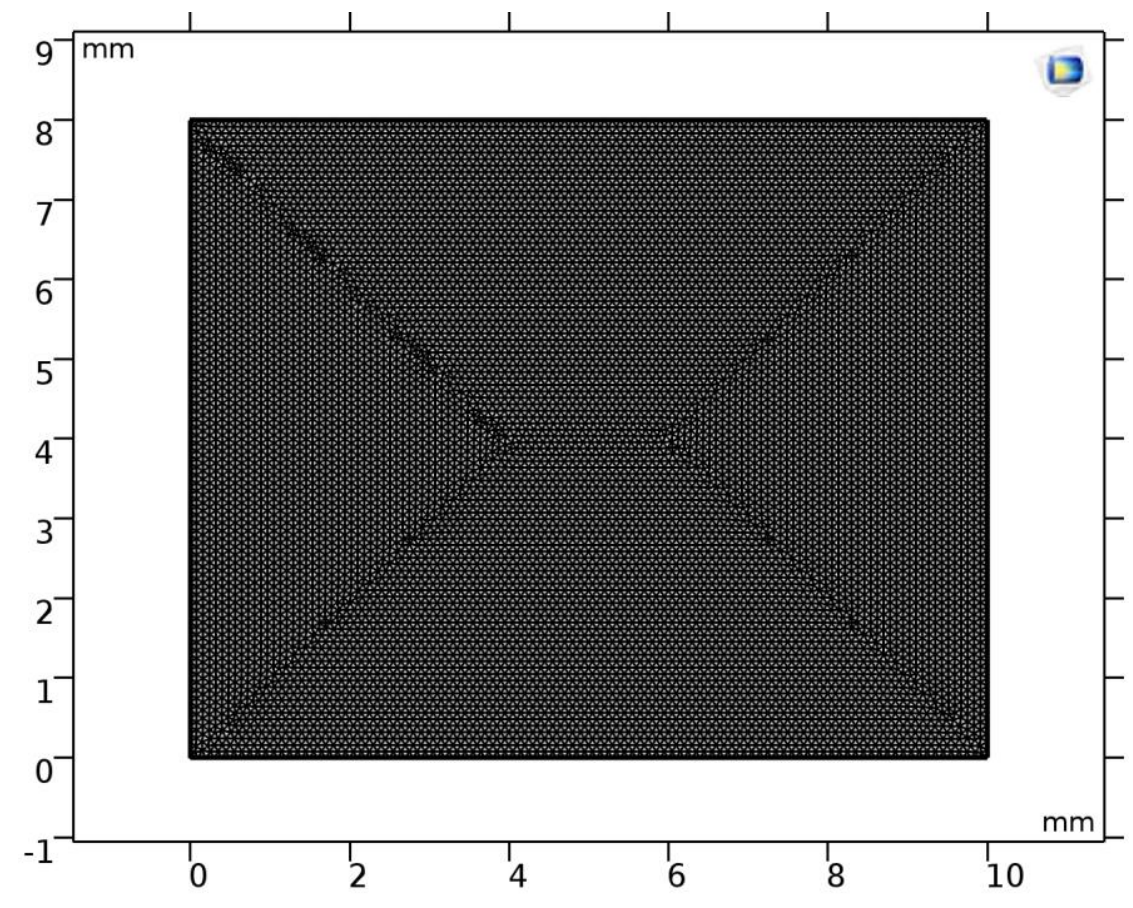

Figure S 1 


\section{Pulse Profile}

The laser pulse profile is as in Figure S 2. FWHMs are $45 \mu \mathrm{m}$ and $1250 \mu \mathrm{m}$ in the directions of $x$ and $y$, respectively. The units of axes are in micrometers.

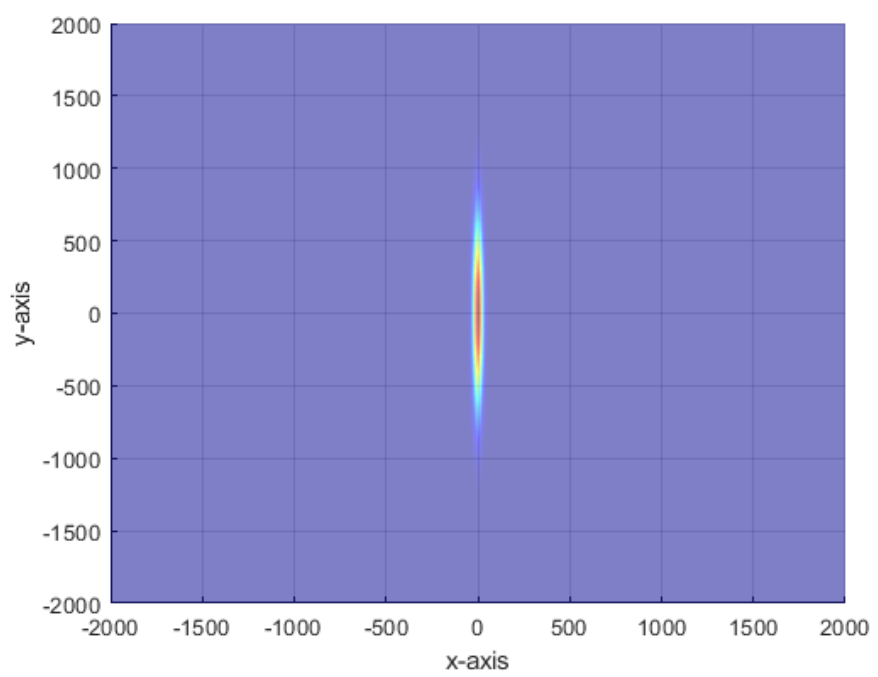

Figure 52

\section{Time Evolution of Pulse}

Figure S3 depicts pulse locations on the surface of the area of interest provided at marked time points. Figure S3 is a representation of Figure $4 b$ in the manuscript at which time mark is " 0 ms" at the moment of laser turn-off. Then the film is let to cool down by heat diffusion. The " $0 \mathrm{~ms}$ " time mark on Figure $4 \mathrm{~b}$ corresponds to time mark of $1000 \mathrm{~ms}$ in Figure 5 of the manuscript.

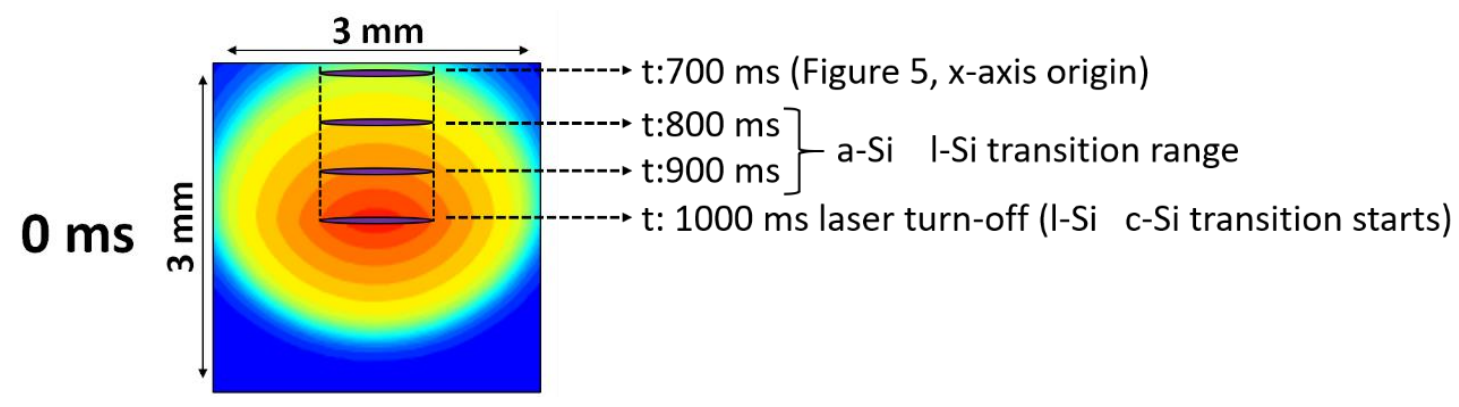

Figure S 3 


\section{Temporal Evolution of Local Temperature}

Our simulation of LC predicts instantaneous temperature gradients reaching astounding values of $10^{6} \mathrm{~K} / \mathrm{s}$ locally (see Figure $\mathrm{S} 4$ ). Due to incomparable mass of substrate vs. film, we expect such deformation to occur at the interface between the film and substrate. This deformation is, however, quenched at the interface and does not propagate into the LC processed film according to secondary ion mass spectroscopy analysis [14, 15], AFM and EBSD measurements.
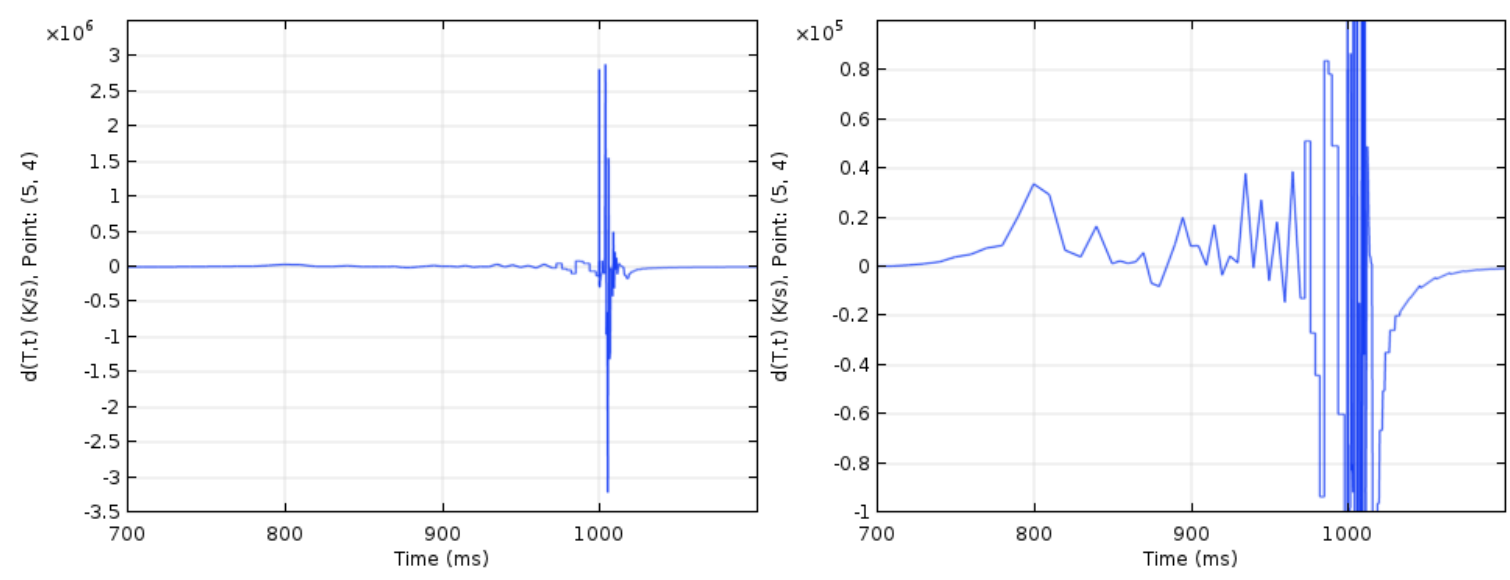

Figure 54

We think that this is an additional benefit of slow quenching operation condition and hence allows LC on substrates at room temperature, unlike in Refs. 4-9. 